\title{
Analysis and Numerical Approximation of an Integro- differential Equation Modeling Non-local Effects in Linear Elasticity
}

\author{
ETIENNE EMMRICH \\ Technische Universität Berlin, Institut für Mathematik, Strasse des 17. Juni 136, 10623 \\ Berlin, Germany \\ OLAF WECKNER \\ Massachusetts Institute of Technology, Department of Mechanical Engineering, Cambridge, \\ MA 02139, USA
}

(Received 4 February 2005; accepted 17 June 2005)

\begin{abstract}
Long-range interactions for linearly elastic media resulting in nonlinear dispersion relations are modeled by an initial-value problem for an integro-differential equation (IDE) that incorporates non-local effects. Interpreting this IDE as an evolutionary equation of second order, well-posedness in $L^{\infty}(\mathbb{R})$ as well as jump relations are proved. Moreover, the construction of the micromodulus function from the dispersion relation is studied. A numerical approximation based upon quadrature is suggested and carried out for two examples, one involving jump discontinuities in the initial data corresponding to a Riemann-like problem.
\end{abstract}

Key Words: Long-range interactions, peridynamic theory, nonlinear dispersion relations, integro-differential equation, existence and uniqueness, jump discontinuity, numerical approximation

\section{INTRODUCTION}

Motivated by the so-called peridynamic theory of solids (see Silling [1], Silling, Zimmermann \& Abeyaratne [2]), we are interested in the integro-differential equation (IDE)

$$
\partial_{t}^{2} u(x, t)=\int_{-\infty}^{\infty} k(x, \xi) u(\xi, t) \mathrm{d} \xi+c(x) u(x, t)+b(x, t), \quad(x, t) \in \mathbb{R} \times(0, T),
$$

where $\mathbb{R}$ denotes the unbounded spatial domain, $(0, T)$ with $T>0$ is the time interval under consideration, $c$ is a time-independent coefficient and $b$ an inhomogeneity. We look for solutions $u=u(x, t)$ to Equation (1.1) that fulfill the initial conditions

$$
u(\cdot, 0)=u_{0}, \quad \partial_{t} u(\cdot, 0)=v_{0}
$$


for prescribed initial data $u_{0}, v_{0}$. In the context of continuum mechanics, $u$ denotes the displacement field whereas $\partial_{t} u$ describes the velocity.

The kernel $k: \mathbb{R}^{2} \rightarrow \mathbb{R}$ might be unsymmetric. However, the integral operator is often to be assumed of convolution type so that

$$
k(x, \xi)=\tilde{k}(x-\xi) \quad \text { with } \quad \tilde{k}(z)=\tilde{k}(-z), \quad z \in \mathbb{R}
$$

and the coefficient $c$ is assumed to be constant and given by

$$
c(x) \equiv-\int_{-\infty}^{\infty} \tilde{k}(z) \mathrm{d} z
$$

The peridynamic theory is a non-local model of integral type, which-in contrast to other such models - only involves the displacement field but not its gradient or higher-order spatial derivatives, as will be discussed in Section 2. This might be an attractive feature, especially in settings that involve singularities. Formally, the peridynamic theory seems to be a continuous version of molecular dynamics; its linearization resembles the equation of motion for a crystalline structure (see Czycholl [3]). However, this similarity is misleading as the peridynamic theory is meant to apply at length scales between those of classical continuum mechanics and molecular dynamics. A discussion of the relation between non-local theories and atomistic models can be found, for example, in Chen et al. [4]. For a detailed description of a variety of non-local theories in continuum mechanics, we refer to Kunin [5] and Rogula [6]. Non-local models can be also found in the description of other natural phenomena. So Lee et al. [7] discuss an IDE of first-order in biomathematics modeling the dynamics of interacting individuals that coexist on a spatial landscape. One of the ideas behind is the description of density changes due to long-distance interactions.

Recently, Weckner and Abeyaratne [8] analysed the effects of long-range forces on the dynamics of an infinite homogeneous bar, corresponding to the IDE

$$
\rho_{0} \partial_{t}^{2} u(x, t)=\int_{-\infty}^{\infty} C(\hat{x}-x)[u(\hat{x}, t)-u(x, t)] \mathrm{d} \hat{x}+h(x, t),(x, t) \in \mathbb{R} \times(0, T),
$$

where $\rho_{0}$ is the constant density and the kernel $C$, the so-called micromodulus function, is of convolution type (1.3) due to the homogeneity. Using Fourier transforms, the authors have shown that the general solution of (1.5) subject to the initial conditions (1.2) can be expressed in terms of a Green function. The motion has been found to be dispersive as a result of the long-range forces. The micromodulus $C$ has been chosen such that the limit of short-range forces is in accordance with the classical results known for a linearly elastic medium. In addition, the first-order correction to classical local elasticity has been derived in order to characterize weak non-locality. The most striking observations made are concerning Riemann's problem with a discontinuous velocity in the beginning: Although the displacement field is initially continuous, a jump discontinuity occurs for all times $t>0$ and remains stationary with respect to the Lagrangian coordinates. Moreover, the height of a displacement jump oscillates around an average value for some materials while for others, it increases monotonically, eventually fracturing the material. 
In this paper, we generalize the equation of motion (1.5) to (1.1) taking into account inhomogeneities in both the inertial and the stiffness distribution resulting in the more general kernel $k$ and coefficient $c$. We prove existence and uniqueness of the mild solution to the initial-value problem for (1.1) in spaces of abstract functions with values in $L^{\infty}(\mathbb{R})$, the space of essentially bounded functions. Because, in the one-dimensional case, any function of bounded variation is also essentially bounded, solvability in $L^{\infty}(\mathbb{R})$ is more general than solvability in the space of functions of bounded variation. As (1.5) is a special case of (1.1), the results here also generalize the uniqueness result given in Weckner and Abeyaratne [8] for a bounded piecewise continuous solution to (1.5) via Fourier transforms.

Moreover, the solution to (1.1) is explicitly given in terms of an infinite series of potencies of a stationary Barbashin operator applied to the initial conditions and the external force field. We also focus on regularity, an a priori estimate for the solution, and stability with respect to the data and the kernel. It is, furthermore, shown that a jump in either the initial conditions or the external force field generates a jump in the displacement field that is stationary with respect to its Lagrangian coordinates. As an example, the Riemann problem is solved. Specializing the results to the homogeneous case, our results agree with those given in [8].

As a closed analytical solution cannot be expected in general, an efficient numerical method has to be developed. For the approximate solution in the case of piecewise continuous data $u_{0}, v_{0}, b$, we suggest a method that is based upon quadrature. For the numerical integration, we partition the domain of integration $\mathbb{R}$ corresponding to the jumps in the data and apply quadrature formulae on each of the integrals taken over intervals on which the data are continuous. The resulting initial value problem for a linear system of second-order differential equations can then be solved by standard methods. Examples are presented in order to illustrate the numerical approximation proposed. The numerical integration is based upon the Gauss-Hermite and composite trapezoidal quadrature.

The paper is organized as follows. In Section 2 we give a short introduction into the peridynamic theory of solids. As a motivation, we present two advantages of the non-local model in comparison to the classical, local model of continuum mechanics and we show how these advantages can be exploited for a number of interesting applications. In Section 3, under certain assumptions on the regularity of the kernel $k$ in (1.1), the mathematical analysis is presented. A numerical approximation is suggested in Section 4. The method proposed is then illustrated in two examples for which analytical solutions are at hand. This allows us to validate the numerical results.

\section{MODELING LONG-RANGE FORCES IN ELASTICITY THEORY}

In the peridynamic theory of a solid (see Silling [1]), the force applied on the particle at position $\vec{x} \in \mathcal{R}$ by the particle at $\overrightarrow{\hat{x}} \in \mathcal{R}$ is characterized by a pairwise force function $\vec{f}=\vec{f}(\overrightarrow{\hat{x}}, \vec{x}, t)$. So the equation of motion for the particle at $\vec{x}$ reads as

$$
\rho_{0}(\vec{x}) \partial_{t}^{2} \vec{u}(\vec{x}, t)=\int_{\mathcal{R}} \vec{f}(\overrightarrow{\hat{x}}, \vec{x}, t) \mathrm{d} \overrightarrow{\hat{x}}+\vec{h}(\vec{x}, t), \quad(\vec{x}, t) \in \mathcal{R} \times(0, T) .
$$


Here, $\mathcal{R} \subseteq \mathbb{R}^{3}$ is the region occupied by the body, $\vec{x}$ is the position of a material point and $\rho_{0}(\vec{x})$ is the mass density at $\vec{x}$, all in the reference configuration. The motion is characterized by the displacement field $\vec{u}=\vec{u}(\vec{x}, t)$ and the material is characterized by $\vec{f}$. The value $\vec{f}(\vec{x}, \vec{x}, t)$ of the pairwise force function denotes the force per unit reference volume applied on the particle at $\vec{x}$ at time $t$ by a unit volume of material at $\overrightarrow{\hat{x}}$. The field $\vec{h}$ represents an external force density.

In the following, we restrict our considerations to the one-dimensional case. Newton's third law requires the symmetry $f(\hat{x}, x, t)=-f(x, \hat{x}, t)$. The constitutive response of the material is characterized by a function $F$, which relates the force between particles to the kinematics. Here, we assume that the relevant variables of the constitutive function are the positions in both the present and the reference configuration:

$$
f(\hat{x}, x, t)=F(y(\hat{x}, t), y(x, t), \hat{x}, x),
$$

where

$$
y(x, t)=x+u(x, t)
$$

is the momentary position of particle $x$ at time $t$ and $u$ is the corresponding displacement. This excludes, for example, materials capable to remember their deformation history. From objectivity principles, the force between the two particles $x$ and $\hat{x}$ cannot depend on the choice of the origin. The constitutive relation is thus given by

$$
F(y(\hat{x}, t), y(x, t), \hat{x}, x)=\tilde{f}(\eta(\hat{x}, x, t), \hat{x}, x), \quad \eta(\hat{x}, x, t):=u(\hat{x}, t)-u(x, t) .
$$

A Taylor expansion with respect to the first argument of the function $\tilde{f}$ leads to

$$
\tilde{f}(\eta(\hat{x}, x, t), \hat{x}, x)=\tilde{f}(0, \hat{x}, x)+\partial_{\eta} \tilde{f}(0, \hat{x}, x) \eta(\hat{x}, x, t)+\mathcal{O}\left(\eta(\hat{x}, x, t)^{2}\right) .
$$

Assuming small relative deformations $\eta$, Equation (2.1) reduces to (1.1) in the onedimensional case with $\mathcal{R}=\mathbb{R}$ and

$$
\begin{aligned}
k(x, \xi) & :=\frac{\partial_{\eta} \tilde{f}(0, \xi, x)}{\rho_{0}(x)}, \quad c(x):=-\int_{-\infty}^{\infty} \frac{\partial_{\eta} \tilde{f}(0, \xi, x)}{\rho_{0}(x)} \mathrm{d} \xi \\
b(x, t) & :=\int_{-\infty}^{\infty} \frac{\tilde{f}(0, \xi, x)}{\rho_{0}(x)} \mathrm{d} \xi+\frac{h(x, t)}{\rho_{0}(x)} .
\end{aligned}
$$

Assuming further a homogeneous material, Equation (1.5) follows.

In what follows, we shall discuss two different applications in which the peridynamic theory seems to be of particular advantage compared to the classical theory of continuum mechanics.

The absence of spatial gradients has been the main motivation for the introduction of the peridynamic theory by Silling [1]. This feature promises computational advantages, especially in settings that involve singularities such as problems arising in fracture mechanics. 
In the classical theory of elasticity, which is based upon partial differential equations, a discontinuity has to be accounted for by dividing the original domain into two subdomains and deriving additional jump conditions. However, discontinuities can form spontaneously and grow along trajectories not known in advance. So the classical method just described seems to be cumbersome for this kind of problem. In the peridynamic theory, the deformation of a body is described by an IDE that remains well-defined both in regular and singular regions of a body. This advantage is currently exploited at a commercial aircraft producer in order to calculate fatigue cracking and failure in composite materials using a code that has been developed by Silling.

The second advantage arises in the modeling of material behavior. The behavior predicted by the peridynamic theory in the case of small wavelengths is quite different from what is predicted by the conventional theory. Especially, the peridynamic theory may imply nonlinear dispersion relations. In contrast, the conventional theory of elasticity predicts linear dispersion curves corresponding to a constant phase velocity. The (nonlinear) dispersion curves can, in principle, be determined by experiments (see Graff [9]). The kernel of the IDE (1.1) can then be constructed from the dispersion relation. The resulting mechanical model contains more information about the material behavior than it can be expressed in conventional local models. In Theorem 3.2, we show that the solution $u_{\varepsilon}$ of the IDE (1.1) with an approximation $k_{\varepsilon}$ of the kernel $k$ is itself an approximation of the original solution $u$. This justifies the construction of $k$ from experimental data.

In the case of a homogeneous, infinite bar, described by the IDE (1.5), a dispersion analysis yields the angular frequency $\omega$ as a function of the wavenumber $k$,

$$
\begin{aligned}
\omega(k) & =\left(\frac{1}{\rho_{0}}(\gamma-\mathcal{F}[C(z)](k))\right)^{1 / 2}=\left(\int_{-\infty}^{\infty}[1-\cos (k z)] \frac{C(z)}{\rho_{0}} \mathrm{~d} z\right)^{1 / 2}, \\
\gamma & :=\mathcal{F}[C(z)](0)=\int_{-\infty}^{\infty} C(z) \mathrm{d} z,
\end{aligned}
$$

where $\mathcal{F}$ denotes the Fourier transform with respect to space. Note that $C(-z)=C(z)$, and so $\omega(-k)=\omega(k)$. The dispersion relation can be used to define the Green function

$$
g(x, t)=\mathcal{F}^{-1}\left[\frac{\sin (\omega(k) t)}{\omega(k)}\right](x, t)=\frac{1}{2 \pi} \int_{-\infty}^{\infty} \cos (k x) \frac{\sin (\omega(k) t)}{\omega(k)} \mathrm{d} k .
$$

The solution to (1.5) is then found by convolution,

$$
\begin{aligned}
u(x, t) & =\int_{-\infty}^{\infty} u_{0}(x-\hat{x}) \partial_{t} g(\hat{x}, t) \mathrm{d} \hat{x}+\int_{-\infty}^{\infty} v_{0}(x-\hat{x}) g(\hat{x}, t) \mathrm{d} \hat{x} \\
& +\int_{0}^{t} \int_{-\infty}^{\infty} \frac{h(x-\hat{x}, t-\hat{t})}{\rho_{0}} g(\hat{x}, \hat{t}) \mathrm{d} \hat{x} \mathrm{~d} \hat{t},
\end{aligned}
$$

which holds in the distributional sense.

On the other hand, the dispersion relation (2.2) can be used to determine the micromodulus function $C$ for a given or experimentally determined dispersion relation $\omega=\omega(k)$. As a 
first example, we assume that, within the relevant range of wavelengths, the measured phase velocity $v_{p}(k)=\omega(k) / k$ is constant such that $v_{p}(k) \equiv c_{0}$, where $c_{0}$ denotes the velocity of sound. This corresponds to the classical linear elasticity theory with a phase velocity that is independent of wavelength. The equation of motion is then given by the wave equation

$$
\rho_{0} \partial_{t}^{2} u(x, t)=E \partial_{x}^{2} u(x, t)+h(x, t)
$$

with Young's modulus $E=\rho_{0} c_{0}^{2}$, which is equivalent to (1.5) with

$$
C(z)=\mathcal{F}^{-1}\left[\gamma-\rho_{0} \omega(k)^{2}\right](z)=\gamma \delta(z)+E \delta^{\prime \prime}(z),
$$

where $\delta=\delta(z)$ denotes Dirac's $\delta$-distribution. In this case, the Green function (2.3) is given by

$$
g(x, t)=\frac{1}{2 c_{0}}\left(H\left(x+c_{0} t\right)-H\left(x-c_{0} t\right)\right),
$$

where $H=H(z)$ denotes HEAVISIDE's jump function that is zero for negative and one for positive arguments. The solution (2.4) of the IDE (1.5) then reduces to the d'Alembert solution of the wave equation.

From an augmented model incorporating lateral contraction, the phase velocity

$$
v_{p}(k)=\frac{c_{0}}{\sqrt{1+(k r v)^{2}}}
$$

results. Here, $v$ is the Poisson number and $r^{2}=J / A$ is the second moment $J$ divided by the cross section area $A$. The equation of motion here reads as

$$
\partial_{t}^{2} u(x, t)=c_{0}^{2} \partial_{x}^{2} u(x, t)+(r v)^{2} \partial_{t}^{2} \partial_{x}^{2} u(x, t) .
$$

Taking into account the additional inertial effect, the improved theory results in a dispersive system extending the range of admissible wavelengths compared to the conventional theory of elasticity where $v=0$. For a detailed discussion, see Graff $[9$, p.121]. However, here we take the point of view that $v_{p}$ is given (for example by the interpolation of experimental data) and the corresponding dynamical model (2.6) is not known in advance. Equation (2.2) can then be used to find the micromodulus function $C$ via $\omega(k)=v_{p}(k) k$ and the experimentally determined $v_{p}$. It follows that

$$
C(z)=\mathcal{F}^{-1}\left[\gamma-\rho_{0}\left(v_{p}(k) k\right)^{2}\right](z)=\gamma \delta(z)+\frac{\rho_{0} c_{0}^{2}}{2 r^{3} v^{3}} \exp \left(-\frac{|z|}{r v}\right) .
$$

So the peridynamic equation of motion reads as

$$
\partial_{t}^{2} u(x, t)=\frac{c_{0}^{2}}{2 r^{3} v^{3}} \int_{-\infty}^{\infty} \exp \left(-\frac{|x-\xi|}{r v}\right) u(\xi, t) \mathrm{d} \xi-\left(\frac{c_{0}}{r v}\right)^{2} u(x, t),
$$


since

$$
\frac{\rho_{0} c_{0}^{2}}{2 r^{3} v^{3}} \int_{-\infty}^{\infty} \exp \left(-\frac{|z|}{r v}\right) \mathrm{d} z=\left(\frac{c_{0}}{r v}\right)^{2}
$$

Indeed, one can show that a smooth, bounded solution to the IDE (2.7) is also a solution to the partial differential equation (2.6) and vice versa, so that both equations are equivalent in the class of sufficiently smooth functions (see also Tricomi [10, p.162]).

These two examples suggest that the same peridynamic equation of motion (1.5) can be used to describe the material behavior at different length scales just by using appropriate micromodulus functions $C$. It, therefore, seems to be promising to model an even more complicated material behavior by means of the peridynamic theory.

\section{MATHEMATICAL ANALYSIS}

As usual, we denote by $\mathcal{C}$ the space of continuous functions, by $\mathcal{C}_{b}$ the space of bounded continuous functions, by $L^{p}(1 \leq p<\infty)$ the space of Lebesgue-measurable functions $u$ such that $|u|^{p}$ is Lebesgue-integrable and by $L^{\infty}$ the space of essentially bounded Lebesguemeasurable functions. The canonical norm in a normed function space $X$ is denoted by $\|\cdot\|_{X}$. Moreover, let $\mathcal{C}^{m}([0, T] ; X)$ with $m \in \mathbb{N}$ be the space of $m$-times continuously differentiable abstract functions $u:[0, T] \rightarrow X$ with norm

$$
\|u\|_{\mathcal{C}^{m}([0, T] ; X)}=\max _{t \in[0, T]} \sum_{j=0}^{m}\left\|\frac{\mathrm{d}^{m} u(t)}{\mathrm{d} t^{m}}\right\|_{X} .
$$

We also write $\mathcal{C}([0, T] ; X)$ if $m=0$. The function space $L^{1}(0, T ; X)$ consists of Bochnerintegrable abstract functions $u:[0, T] \rightarrow X$ such that $t \mapsto\|u(t)\|_{X}$ is Lebesgue-integrable and is equipped with the norm

$$
\|u\|_{L^{1}(0, T ; X)}=\int_{0}^{T}\|u(t)\| \mathrm{d} t .
$$

Unfortunately, we cannot apply the Hilbert-Schmidt theory as the integral operator might be of convolution type and the solutions to (1.1) we have in mind are not integrable over the whole line $(-\infty, \infty)$.

For the kernel $k$, we shall assume

(H1) $k \in \mathcal{C}(\mathbb{R} \times \mathbb{R})$ with $k_{0}:=\sup _{x \in \mathbb{R}} \int_{-\infty}^{\infty}|k(x, \xi)| \mathrm{d} \xi<\infty$;

(H2) for all $x \in \mathbb{R}$ and $\varepsilon>0$ there is a $\delta>0$ such that for all $y \in \mathbb{R}$

$$
|x-y|<\delta \text { implies } \int_{-\infty}^{\infty}|k(x, \xi)-k(y, \xi)| \mathrm{d} \xi<\varepsilon .
$$


Note that, for convolution operators, $(\mathrm{H} 1)$ is fulfilled if $\tilde{k} \in \mathcal{C}(\mathbb{R}) \cap L^{1}(\mathbb{R})$. We then have $k_{0}=\|\tilde{k}\|_{L^{1}(\mathbb{R})}$. Assumption (H2) does not follow from the continuity of $k$ as the domain is unbounded. However, it is fulfilled if $k$ possesses a partial derivative $k_{x}=k_{x}(x, \xi)$ such that for all $x \in \mathbb{R}$

$$
\sup _{x \in \mathbb{R}} \int_{-\infty}^{\infty}\left|k_{x}(x, \xi)\right| \mathrm{d} \xi<\infty
$$

or if $k$ has compact support on $\mathbb{R} \times \mathbb{R}$ (which cannot be the case for convolution operators).

\section{Example 3.1. For}

$$
\tilde{k}(z)=\left\{\begin{array}{cl}
\frac{\sin z-z \cos z}{z^{3}} & \text { if } z \neq 0, \\
\frac{1}{3} & \text { if } z=0,
\end{array} \quad k(x, \xi)=\tilde{k}(x-\xi),\right.
$$

the assumptions (H1) and (H2) are fulfilled. Moreover,

$$
c(x) \equiv-\int_{-\infty}^{\infty} \tilde{k}(z) \mathrm{d} z=-\pi
$$

see also Example 4.2 in Section 4.

Let $c \in L^{\infty}(\mathbb{R})$. For functions $u=u(x) \in L^{\infty}(\mathbb{R})$, we define the (autonomous) operator $A$ via

$$
(A u)(x):=\int_{-\infty}^{\infty} k(x, \xi) u(\xi) \mathrm{d} \xi+c(x) u(x), \quad x \in \mathbb{R}
$$

Due to (H1), the operator $A$ maps into $L^{\infty}(\mathbb{R})$ and is linear and bounded with

$$
\|A u\|_{L^{\infty}(\mathbb{R})} \leq \alpha\|u\|_{L^{\infty}(\mathbb{R})}, \quad \alpha:=k_{0}+\|c\|_{L^{\infty}(\mathbb{R})}
$$

We may write $A$ as the sum of the integral and the multiplication operator, such that

$$
A=K+C, \text { where }(K u)(x):=\int_{-\infty}^{\infty} k(x, \xi) u(\xi) \mathrm{d} \xi,(C u)(x):=c(x) u(x), x \in \mathbb{R}
$$

So $A$ is a stationary Barbashin operator (see Appell et al. [11]).

Proposition 3.1. Under the assumptions $(\mathrm{H} 1)$ and $(\mathrm{H} 2)$, the integral operator $K$ maps $u \in$ $L^{\infty}(\mathbb{R})$ to the bounded continuous function $K u \in \mathcal{C}_{b}(\mathbb{R})$.

Let $c$ be continuous. If $u \in L^{\infty}(\mathbb{R})$ is continuous then $A u$ is continuous, too. If $u \in$ $L^{\infty}(\mathbb{R})$ is a piecewise continuous function with a jump at $\hat{x}$ of height 


$$
\llbracket u(\hat{x}) \rrbracket:=\lim _{x \searrow \hat{x}} u(x)-\lim _{x>\hat{x}} u(x)
$$

then $A u$ is again piecewise continuous with a jump at $\hat{x}$ of height

$$
\llbracket(A u)(\hat{x}) \rrbracket=c(\hat{x}) \llbracket u(\hat{x}) \rrbracket .
$$

Proof. Let $u \in L^{\infty}(\mathbb{R})$ be arbitrary. We show that $K u$ is continuous on $\mathbb{R}$. For $x, y \in \mathbb{R}$, we have

$$
\begin{aligned}
|(K u)(x)-(K u)(y)| & =\left|\int_{-\infty}^{\infty}(k(x, \xi)-k(y, \xi)) u(\xi) \mathrm{d} \xi\right| \\
& \leq \int_{-\infty}^{\infty}|k(x, \xi)-k(y, \xi)| \mathrm{d} \xi\|u\|_{L^{\infty}(\mathbb{R})} .
\end{aligned}
$$

Because of (H1), it holds that

$$
\int_{-\infty}^{\infty}|k(x, \xi)-k(y, \xi)| \mathrm{d} \xi \leq 2 k_{0},
$$

so the integral converges for all $x, y \in \mathbb{R}$. The continuity of $K u$ follows from (H2). Moreover, we find from (H1) that

$$
|(K u)(x)| \leq \int_{\infty}^{\infty}|k(x, \xi)| \mathrm{d} \xi\|u\|_{L^{\infty}(\mathbb{R})} \leq k_{0}\|u\|_{L^{\infty}(\mathbb{R})}
$$

for all $x \in \mathbb{R}$, so $K u$ is bounded.

If $c$ and $u$ are continuous then $c u$ is continuous, too. Hence $A u=K u+C u$ is continuous. If $c$ is continuous and $u$ has a jump at $\hat{x}$ then $K u$ is continuous at $\hat{x}$ and $(C u)(x)=c(x) u(x)$ has a jump at $\hat{x}$ of height $c(\hat{x}) \llbracket u(\hat{x}) \rrbracket$. Since $A u=K u+C u$, the assertion follows.

Denoting by $u=u(t)$ and $b=b(t)$ the abstract functions with values in $L^{\infty}(\mathbb{R})$ that correspond to $u=u(x, t)$ and $b=b(x, t)$, respectively, we may consider the evolutionary equation

$$
\ddot{u}(t)=A u(t)+b(t), \quad t \in(0, T),
$$

instead of (1.1), where $\ddot{u}$ is the second derivative of $u:[0, T] \rightarrow L^{\infty}(\mathbb{R})$.

On the product space $L^{\infty}(\mathbb{R}) \times L^{\infty}(\mathbb{R})$, equipped with the norm

$$
\left\|(u, v)^{\mathrm{T}}\right\|:=\max \left\{\|u\|_{L^{\infty}(\mathbb{R})},\|v\|_{L^{\infty}(\mathbb{R})}\right\},
$$

we can now define the operator 


$$
\mathcal{A}:=\left(\begin{array}{cc}
0 & I \\
A & 0
\end{array}\right), \quad \mathcal{A}\left(\begin{array}{l}
u \\
v
\end{array}\right)=\left(\begin{array}{c}
v \\
A u
\end{array}\right)
$$

where $I$ denotes the identity in $L^{\infty}(\mathbb{R})$. The operator $\mathcal{A}: L^{\infty}(\mathbb{R})^{2} \rightarrow L^{\infty}(\mathbb{R})^{2}$ is linear and bounded with

$$
\left\|\mid \mathcal{A}\left(\begin{array}{l}
u \\
v
\end{array}\right)\right\| \leq \hat{\alpha}\left\|\left(\begin{array}{l}
u \\
v
\end{array}\right)\right\|, \quad \hat{\alpha}:=\max \{1, \alpha\} .
$$

With $v:=\dot{u}$, Equation (3.2) is equivalent to the first-order operator-differential equation

$$
\frac{\mathrm{d}}{\mathrm{d} t}\left(\begin{array}{l}
u(t) \\
v(t)
\end{array}\right)=\mathcal{A}\left(\begin{array}{l}
u(t) \\
v(t)
\end{array}\right)+\left(\begin{array}{c}
0 \\
b(t)
\end{array}\right), \quad t \in(0, T) .
$$

Because $\mathcal{A}$ is a linear bounded operator, there immediately follows, from standard arguments and Duhamel's principle (see e.g. Amann [12, pp. 151 ff.] or Emmrich [13, pp. 173 ff.]), the following proposition.

Proposition 3.2. Under the assumption (H1), the operator $\mathcal{A}: L^{\infty}(\mathbb{R})^{2} \rightarrow L^{\infty}(\mathbb{R})^{2}$ generates the uniformly continuous semigroup $\left\{\mathrm{e}^{t \mathcal{A}}\right\}_{t \geq 0}$ with

$$
\mathrm{e}^{t \mathcal{A}}=\left(\begin{array}{cc}
\cosh (t \sqrt{A}) & \sqrt{A}^{-1} \sinh (t \sqrt{A}) \\
\sqrt{A} \sinh (t \sqrt{A}) & \cosh (t \sqrt{A})
\end{array}\right)=\left(\begin{array}{cc}
\sum_{n=0}^{\infty} \frac{t^{2 n} A^{n}}{(2 n) !} & \sum_{n=0}^{\infty} \frac{t^{2 n+1} A^{n}}{(2 n+1) !} \\
\sum_{n=0}^{\infty} \frac{t^{2 n+1} A^{n+1}}{(2 n+1) !} & \sum_{n=0}^{\infty} \frac{t^{2 n} A^{n}}{(2 n) !}
\end{array}\right)
$$

Let $b \in L^{1}\left(0, T ; L^{\infty}(\mathbb{R})\right)$. Then

$$
\left(\begin{array}{l}
u(t) \\
v(t)
\end{array}\right)=\mathrm{e}^{t \mathcal{A}}\left(\begin{array}{l}
u_{0} \\
v_{0}
\end{array}\right)+\int_{0}^{t} \mathrm{e}^{(t-s) \mathcal{A}}\left(\begin{array}{c}
0 \\
b(s)
\end{array}\right) \mathrm{d} s, \quad t \in(0, T),
$$

is the unique mild solution to (3.4) with the initial values $u(0)=u_{0} \in L^{\infty}(\mathbb{R})$ and $v(0)=$ $v_{0} \in L^{\infty}(\mathbb{R})$. If $b \in \mathcal{C}\left([0, T] ; L^{\infty}(\mathbb{R})\right)$ then $\ddot{u} \in \mathcal{C}\left([0, T] ; L^{\infty}(\mathbb{R})\right)$.

Note that $\sqrt{A}$ and $\sqrt{A}^{-1}$ is only a symbolic notation as $A$ need not to be invertible but $\cosh (t \sqrt{A}), \sqrt{A}^{-1} \sinh (t \sqrt{A})$, and $\sqrt{A} \sinh (t \sqrt{A})$ are well defined as series of potencies of $A$ as in (3.5). The representation (3.5) follows from the special form of $\mathcal{A}$ given in (3.3).

Theorem 3.1. Let $b \in L^{1}\left(0, T ; L^{\infty}(\mathbb{R})\right)$ and assume $(H 1)$. Then

$$
u(t)=\cosh (t \sqrt{A}) u_{0}+\sqrt{A}^{-1} \sinh (t \sqrt{A}) v_{0}+\sqrt{A}^{-1} \int_{0}^{t} \sinh ((t-s) \sqrt{A}) b(s) \mathrm{d} s
$$

is the unique mild solution to (3.2) with the initial values $u(0)=u_{0} \in L^{\infty}(\mathbb{R})$ and $\dot{u}(0)=$ $v_{0} \in L^{\infty}(\mathbb{R})$ and lies in $C\left([0, T] ; L^{\infty}(\mathbb{R})\right)$. Moreover, the a priori estimate 


$$
\begin{aligned}
\|u(t)\|_{L^{\infty}(\mathbb{R})} & \leq \cosh (t \sqrt{\alpha})\left\|u_{0}\right\|_{L^{\infty}(\mathbb{R})}+\sqrt{\alpha}^{-1} \sinh (t \sqrt{\alpha})\left\|v_{0}\right\|_{L^{\infty}(\mathbb{R})} \\
& +\sqrt{\alpha}^{-1} \int_{0}^{t} \sinh ((t-s) \sqrt{\alpha})\|b(s)\|_{L^{\infty}(\mathbb{R})} \mathrm{d} s
\end{aligned}
$$

holds for $t \in(0, T)$. If $b \in \mathcal{C}^{m}\left([0, T] ; L^{\infty}(\mathbb{R})\right)(m \in \mathbb{N})$ then $u \in \mathcal{C}^{m+2}\left([0, T] ; L^{\infty}(\mathbb{R})\right)$. If $b$ is constant in time, then

$$
u(t)=\cosh (t \sqrt{A}) u_{0}+\sqrt{A}^{-1} \sinh (t \sqrt{A}) v_{0}+A^{-1}(\cosh (t \sqrt{A})-I) b
$$

Proof. Existence and uniqueness follow directly from Proposition 3.2. The estimate (3.8) follows from the boundedness of $A$ and properties of the Bochner integral. The representation (3.7) results from (3.5) and (3.6). The regularity is a direct consequence of the differential equation (3.2) and the linearity of the time-independent operator $A$. Carrying out the integration gives the representation for stationary $b$ with the symbolic notation

$$
A^{-1}(\cosh (t \sqrt{A})-I):=\sum_{n=1}^{\infty} \frac{t^{2 n} A^{n-1}}{(2 n) !} .
$$

In virtue of the linearity of the problem, the estimate (3.8) also proves stability with respect to the data $u_{0}, v_{0}$, and $b$. If $u_{1}$ and $u_{2}$ are the solutions with respect to the data $u_{01}, v_{01}, b_{1}$ and $u_{02}, v_{02}, b_{2}$, respectively, then $u_{1}-u_{2}$ is the solution with respect to the data $u_{01}-u_{02}, v_{01}-v_{02}, b_{1}-b_{2}$. There then follows from (3.8) the stability estimate

$$
\begin{aligned}
& \left\|u_{1}(t)-u_{2}(t)\right\|_{L^{\infty}(\mathbb{R})} \\
\leq & \cosh (t \sqrt{\alpha})\left\|u_{01}-u_{02}\right\|_{L^{\infty}(\mathbb{R})}+\sqrt{\alpha}^{-1} \sinh (t \sqrt{\alpha})\left\|v_{01}-v_{02}\right\|_{L^{\infty}(\mathbb{R})} \\
+ & \sqrt{\alpha}^{-1} \int_{0}^{t} \sinh ((t-s) \sqrt{\alpha})\left\|b_{1}(s)-b_{2}(s)\right\|_{L^{\infty}(\mathbb{R})} \mathrm{d} s .
\end{aligned}
$$

Theorem 3.2. Let $b \in L^{1}\left(0, T ; L^{\infty}(\mathbb{R})\right)$ and let, for arbitrary $\varepsilon>0, k$ and $k_{\varepsilon}$ be kernels satisfying $(H 1)$ and $c, c_{\varepsilon} \in L^{\infty}(\mathbb{R})$ such that

$$
\sup _{x \in \mathbb{R}} \int_{-\infty}^{\infty}\left|k(x, \xi)-k_{\varepsilon}(x, \xi)\right| \mathrm{d} \xi+\left\|c-c_{\varepsilon}\right\|_{L^{\infty}(\mathbb{R})}<\varepsilon .
$$

For the unique mild solutions $u, u_{\varepsilon} \in \mathcal{C}\left([0, T] ; L^{\infty}(\mathbb{R})\right)$ to Equation (3.2) with $k$, $c$ and $k_{\varepsilon}$, $c_{\varepsilon}$, respectively, and with the initial values $u(0)=u_{\varepsilon}(0)=u_{0} \in L^{\infty}(\mathbb{R})$ and $\dot{u}(0)=\dot{u}_{\varepsilon}(0)=$ $v_{0} \in L^{\infty}(\mathbb{R})$, the estimate

$$
\left\|u(t)-u_{\varepsilon}(t)\right\|_{L^{\infty}(\mathbb{R})} \leq \varepsilon \sqrt{\alpha+\varepsilon}^{-1} \int_{0}^{t} \sinh ((t-s) \sqrt{\alpha+\varepsilon})\|u(s)\|_{L^{\infty}(\mathbb{R})} \mathrm{d} s
$$

holds true for all $t \in(0, T)$. 
Proof. Let $A_{\varepsilon}$ be the operator (3.1) corresponding to $k_{\varepsilon}$ and $c_{\varepsilon}$. For the difference $w_{\varepsilon}:=u-$ $u_{\varepsilon}$, it follows that

$$
\ddot{w}_{\varepsilon}=A_{\varepsilon} w_{\varepsilon}+\left(A-A_{\varepsilon}\right) u
$$

with $w_{\varepsilon}(0)=\dot{w}_{\varepsilon}(0)=0$. Note that $\left(A-A_{\varepsilon}\right) u \in \mathcal{C}\left([0, T] ; L^{\infty}(\mathbb{R})\right)$ since $u \in \mathcal{C}([0, T]$; $L^{\infty}(\mathbb{R})$ ) and $A-A_{\varepsilon}$ is a linear bounded operator in $L^{\infty}(\mathbb{R})$. Due to Theorem 3.1, now applied on the foregoing initial-value problem with the unknown $w_{\varepsilon}$ and the inhomogeneity $\left(A-A_{\varepsilon}\right) u$, and because of

$$
\left\|A_{\varepsilon} v\right\|_{L^{\infty}(\mathbb{R})} \leq\|A v\|_{L^{\infty}(\mathbb{R})}+\left\|A v-A_{\varepsilon} v\right\|_{L^{\infty}(\mathbb{R})} \leq \alpha\|v\|_{L^{\infty}(\mathbb{R})}+\varepsilon\|v\|_{L^{\infty}(\mathbb{R})}
$$

for all $v \in L^{\infty}(\mathbb{R})$, we find

$$
\left\|w_{\varepsilon}(t)\right\|_{L^{\infty}(\mathbb{R})} \leq \sqrt{\alpha+\varepsilon}^{-1} \int_{0}^{t} \sinh ((t-s) \sqrt{\alpha+\varepsilon})\left\|\left(A-A_{\varepsilon}\right) u(s)\right\|_{L^{\infty}(\mathbb{R})} \mathrm{d} s
$$

and thus the assertion.

Estimate (3.10), together with (3.8), justifies the idea of measuring the dispersion relation and the experimental determination of the micromodulus function, which then gives the kernel $k$ and the coefficient $c$ : If $k_{\varepsilon}$ is an approximation of $k$ than $u_{\varepsilon}$ is an approximation of $u$, too.

Corollary 3.1. Let $b \in L^{1}\left(0, T ; L^{\infty}(\mathbb{R})\right)$ and assume $(\mathrm{H} 1)$ and $(\mathrm{H} 2)$ with $c \in \mathcal{C}_{b}(\mathbb{R})$. If $u_{0}, v_{0}$ and $b(t)(t \in[0, T])$ are bounded and continuous then the solution $u$ from Theorem 3.1, given by (3.7), is in $\mathcal{C}([0, T] \times \mathbb{R})$. If, in addition, $b \in \mathcal{C}\left([0, T] ; \mathcal{C}_{b}(\mathbb{R})\right)$ then $u$ is the unique classical solution to the initial-value problem for (1.1). If $u_{0}, v_{0}$ or the stationary $b$ is bounded and piecewise continuous with a jump at $\hat{x}$ then the solution $u$ is also piecewise continuous with respect to $x$ and, for all times $t \in[0, T]$, the jump remains at $\hat{x}$ with

$$
\begin{aligned}
\llbracket u(\hat{x}, t) \rrbracket & =\cosh (t \sqrt{c(\hat{x})}) \llbracket u_{0}(\hat{x}) \rrbracket+\sqrt{c(\hat{x})}^{-1} \sinh (t \sqrt{c(\hat{x})}) \llbracket v_{0}(\hat{x}) \rrbracket \\
& +c(\hat{x})^{-1}(\cosh (t \sqrt{c(\hat{x})})-1) \llbracket b(\hat{x}) \rrbracket
\end{aligned}
$$

and no other jumps are generated. If $c \equiv 0$ then

$$
\llbracket u(\hat{x}, t) \rrbracket=\llbracket u_{0}(\hat{x}) \rrbracket+t \llbracket v_{0}(\hat{x}) \rrbracket+\frac{1}{2} t^{2} \llbracket b(\hat{x}) \rrbracket .
$$

Proof. From Proposition 3.1, we know that $A$ maps continuous functions into continuous functions. Potencies of $A$ do so, too. The solution $u \in \mathcal{C}\left([0, T] ; L^{\infty}(\mathbb{R})\right)$ from Theorem 3.1 is, therefore, continuous with respect to $x$ if $u_{0}, v_{0}$ and $b(t)(t \in[0, T])$ are continuous. It follows the continuity on $[0, T] \times \mathbb{R}$. If $b \in \mathcal{C}\left([0, T] ; \mathcal{C}_{b}(\mathbb{R})\right)$ then $u \in \mathcal{C}^{2}\left([0, T] ; \mathcal{C}_{b}(\mathbb{R})\right)$ and $u$ solves (1.1) in the classical sense. Moreover, (1.1) possesses at most one solution since any 
classical solution is also a mild solution but the mild solution is unique. The jump relations immediately follow from Proposition 3.1 and the representation (3.9).

The jump relation above is in agreement with Weckner and Abeyaratne [8], who proved the stationarity of jumps in solutions to (1.5).

Example 3.2. Consider the Riemann-like problem with $c(x) \equiv c \in \mathbb{R}, u_{0}(x)=u_{l} H(-x)+$ $u_{r} H(x)\left(u_{l}, u_{r} \in \mathbb{R}\right.$ given $), v_{0}(x) \equiv 0$, and $b(x, t) \equiv 0$, where $H$ denotes again the Heaviside function. Then

$$
u(x, t)=\sum_{n=0}^{\infty} \frac{t^{2 n}}{(2 n) !}\left(A^{n} u_{0}\right)(x)
$$

with

$$
\llbracket u(0, t) \rrbracket=\left(u_{r}-u_{l}\right) \sum_{n=0}^{\infty} \frac{t^{2 n} c^{n}}{(2 n) !}=\left(u_{r}-u_{l}\right) \cdot\left\{\begin{array}{cl}
\cosh (t \sqrt{c}) & \text { if } c>0 \\
1 & \text { if } c=0 \\
\cos (t \sqrt{-c}) & \text { if } c<0
\end{array}\right.
$$

Note that a jump in the initial velocity $v_{0}$ produces a jump in the displacement field $u=u(x, t)$ for $t>0$ even if the initial displacement $u_{0}$ is continuous. This is shown in the following example.

Example 3.3. Consider the Riemann-like problem with $c(x) \equiv c \in \mathbb{R}, u_{0}(x) \equiv 0, v_{0}(x)=$ $v_{l} H(-x)+v_{r} H(x)\left(v_{l}, v_{r} \in \mathbb{R}\right.$ given $)$, and $b(x, t) \equiv 0$. Then

$$
u(x, t)=\sum_{n=0}^{\infty} \frac{t^{2 n+1}}{(2 n+1) !}\left(A^{n} v_{0}\right)(x)
$$

with

$$
\llbracket u(0, t) \rrbracket=\left(v_{r}-v_{l}\right) \sum_{n=0}^{\infty} \frac{t^{2 n+1} c^{n}}{(2 n+1) !}=\left(v_{r}-v_{l}\right) \cdot\left\{\begin{array}{cl}
\frac{1}{\sqrt{c}} \sinh (t \sqrt{c}) & \text { if } c>0 \\
t & \text { if } c=0 \\
\frac{1}{\sqrt{-c}} \sin (t \sqrt{-c}) & \text { if } c<0 .
\end{array}\right.
$$

\section{NUMERICAL APPROXIMATION AND EXAMPLES}

For the numerical solution of (1.1), we suggest the following method. We assume that $u_{0}$, $v_{0}$, and $b(\cdot, t)(t \in[0, T])$ are bounded and piecewise continuous and that for any fixed $x$ the mapping $t \mapsto b(x, t)$ is continuous. Let 


$$
-\infty<s_{1}<s_{2}<\cdots<s_{m}<\infty
$$

be the abscissae at which $u_{0}, v_{0}$ or $b(\cdot, t)$ has a jump discontinuity. The integral operator $K$ may then be represented as

$$
\begin{aligned}
(K u)(x) & =\int_{-\infty}^{s_{1}} k(x, \xi) u(\xi) \mathrm{d} \xi+\int_{s_{1}}^{s_{2}} k(x, \xi) u(\xi) \mathrm{d} \xi+\cdots+\int_{s_{m}}^{\infty} k(x, \xi) u(\xi) \mathrm{d} \xi \\
& =: \quad\left(K_{0} u\right)(x)+\left(K_{1} u\right)(x)+\cdots+\left(K_{m} u\right)(x) .
\end{aligned}
$$

If $m=0$ then $K \equiv K_{0}$. For each integral operator $K_{i}(i=0,1, \ldots, m)$, we consider a sequence of quadrature formulae

$$
\left(K_{i} u\right)(x) \approx \sum_{j=0}^{N_{i}} \sigma_{i, j}^{\left(N_{i}\right)} k\left(x, \xi_{i, j}^{\left(N_{i}\right)}\right) u\left(\xi_{i, j}^{\left(N_{i}\right)}\right)=:\left(K_{i}^{\left(N_{i}\right)} u\right)(x)
$$

where $N_{i} \in \mathbb{N}$, the weights $\sigma_{i, j}^{\left(N_{i}\right)}$, and the quadrature points $\xi_{i, j}^{\left(N_{i}\right)}$ are given. Note that $\xi_{i, N_{i}}^{\left(N_{i}\right)}$ and $\xi_{i+1,0}^{\left(N_{i+1}\right)}$ might coincide. For convenience, we introduce another notation:

$N:=\sum_{i=0}^{m}\left(N_{i}+1\right)-1, \quad x_{n}^{(N)}:=\xi_{i, j}^{\left(N_{i}\right)}, \quad \sigma_{n}^{(N)}:=\sigma_{i, j}^{\left(N_{i}\right)} \quad$ with $\quad n=\sum_{\nu=0}^{i-1}\left(N_{\nu}+1\right)+j$

We shall assume

$$
x_{0}^{(N)} \leq x_{1}^{(N)} \leq \cdots \leq x_{N}^{(N)}
$$

The integral operator $K$ is thus approximated by the composite quadrature

$$
(K u)(x) \approx \sum_{n=0}^{N} \sigma_{n}^{(N)} k\left(x, x_{n}^{(N)}\right) u\left(x_{n}^{(N)}\right)=:\left(K^{(N)} u\right)(x) .
$$

Regarding the IDE (1.1), remember that its solution $u=u(x, t)$ possesses only jumps at the abscissae $s_{1}, \ldots, s_{m}$, which justifies the composite quadrature suggested. We are now looking for functions $U_{l}^{(N)}=U_{l}^{(N)}(t)$ approximating $t \mapsto u\left(x_{l}^{(N)}, t\right)(l=0,1, \ldots, N)$ that fulfill the initial-value problem

$$
\begin{gathered}
\frac{\mathrm{d}^{2} U_{l}^{(N)}(t)}{\mathrm{d} t^{2}}=\sum_{n=0}^{N} \sigma_{n}^{(N)} k\left(x_{l}^{(N)}, x_{n}^{(N)}\right) U_{n}^{(N)}(t)+c\left(x_{l}^{(N)}\right) U_{l}^{(N)}(t)+b\left(x_{l}^{(N)}, t\right), \\
l=0,1, \ldots, N, t \in(0, T)
\end{gathered}
$$

with 


$$
U_{l}^{(N)}(0)=u_{0}\left(x_{l}^{(N)}\right), \quad \frac{\mathrm{d} U_{l}^{(N)}(0)}{\mathrm{d} t}=v_{0}\left(x_{l}^{(N)}\right), \quad l=0,1, \ldots, N .
$$

As this is a linear system, there is a unique solution $U^{(N)}:=\left(U_{0}^{(N)}, \ldots, U_{N}^{(N)}\right)^{\mathrm{T}}$.

In order to demonstrate the numerical method presented we consider two examples for the solution to the initial-value problem for the IDE (1.5) of the homogeneous bar. For both examples, analytical solutions are at hand, which allows us to validate our numerical results and also to discuss the limits of the method suggested. The first example shows the response to continuous initial conditions while the second example is concerned with discontinuous initial conditions: the velocity field involves jump discontinuities and the initial displacement field is taken to be zero.

Example 4.1 (Gaussian initial displacement). We consider (1.5) for the initial conditions and micromodulus function

$$
u_{0}(x)=U \mathrm{e}^{-(x / L)^{2}}, \quad v_{0}(x) \equiv 0, \quad C(x)=\frac{4 E}{\ell^{3} \sqrt{\pi}} \mathrm{e}^{-(x / \ell)^{2}}
$$

with Young's modulus $E$, a length-scale parameter $\ell>0$, and $U \in \mathbb{R}, L>0$ given. For $\ell \rightarrow 0$, equation (1.5) then becomes the wave equation of local elasticity theory

$$
\rho_{0} \partial_{t}^{2} u(x, t)=E \partial_{x}^{2} u(x, t)+h(x, t) .
$$

So $\ell$ represents the degree of non-locality. In the following, let the right-hand side $h$ be zero. With the normalization

$$
\hat{x}:=\frac{x}{L}, \quad \hat{t}:=\frac{c_{0} t}{L} \quad \text { with } \quad c_{0}:=\sqrt{\frac{E}{\rho_{0}}}, \quad \hat{u}(\hat{x}, \hat{t}):=\frac{u(x, t)}{U},
$$

the equation of motion (1.5) can be rewritten in dimensionless form (1.1) with the convolution kernel $\tilde{k}$ and the constant coefficient $c$ given by

$$
\tilde{k}(\hat{z}):=\frac{L^{3}}{E} C(z)=\frac{4}{\sqrt{\pi}}\left(\frac{L}{\ell}\right)^{3} \mathrm{e}^{-(\hat{z} L / \ell)^{2}}, \quad c(\hat{x}):=-\int_{-\infty}^{\infty} \tilde{k}(\hat{z}) \mathrm{d} \hat{z}=-\left(\frac{2 L}{\ell}\right)^{2} .
$$

The exact solution of this initial-value problem is given by

$$
\hat{u}(\hat{x}, \hat{t})=\frac{2}{\sqrt{\pi}} \int_{0}^{\infty} \cos (2 \alpha \hat{x}) \mathrm{e}^{-\alpha^{2}} \cos \left(\frac{2 L \hat{t}}{\ell} \sqrt{1-\mathrm{e}^{-(\alpha \ell / L)^{2}}}\right) \mathrm{d} \alpha,
$$

see Weckner \& Abeyaratne [8]. Expanding (4.5) in a Taylor series around $\ell / L=0$, one obtains in the limit $\ell / L \rightarrow 0$ the d'Alembert solution to (4.4) 


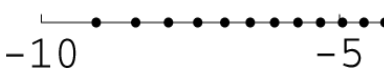

Figure 1. Roots of the Hermite polynomial $H_{49}$.

$$
(\hat{x}, \hat{t}) \mapsto U \frac{\mathrm{e}^{-(\hat{x}-\hat{t})^{2}}+\mathrm{e}^{-(\hat{x}+\hat{t})^{2}}}{2} .
$$

In what follows, we omit the hat and deal only with the dimensionless quantities.

We apply the numerical method suggested with $m=0$ as there is no discontinuity in the data. The numerical integration is based upon the Gauss-Hermite quadrature

$$
\int_{-\infty}^{\infty} \mathrm{e}^{-\xi^{2}} \Phi(\xi) \mathrm{d} \xi \approx \sum_{j=0}^{N} \gamma_{j}^{(N)} \Phi\left(\xi_{j}^{(N)}\right)
$$

where $\xi_{0}^{(N)}<\xi_{1}^{(N)}<\cdots<\xi_{N}^{(N)}$ are the roots of the Hermite polynomial

$$
H_{N+1}(\xi):=(-1)^{N+1} \mathrm{e}^{\xi^{2}} \frac{\mathrm{d}^{N+1}}{\mathrm{~d} \xi^{N+1}}\left(\mathrm{e}^{-\xi^{2}}\right)
$$

and the weights $\gamma_{j}^{(N)}>0(j=0,1, \ldots, N)$ are given by

$$
\gamma_{j}^{(N)}:=\int_{-\infty}^{\infty} \mathrm{e}^{-\xi^{2}} L_{j}^{(N)}(\xi) \mathrm{d} \xi, \quad L_{j}^{(N)}(\xi):=\prod_{\substack{i=0 \\ i \neq j}}^{N} \frac{\xi-\xi_{i}^{(N)}}{\xi_{j}^{(N)}-\xi_{i}^{(N)}} .
$$

The Gauss-Hermite quadrature is exact for polynomials $\Phi=\Phi(\xi)$ of highest degree $2 N+1$.

The approximate operator $K^{(N)}$ is now given by (4.1) with

$$
\sigma_{j}^{(N)}:=\gamma_{j}^{(N)} \exp \left(\left(\xi_{j}^{(N)}\right)^{2}\right), \quad x_{j}^{(N)}:=\xi_{j}^{(N)} \quad(j=0,1, \ldots, N),
$$

and we have to solve the initial-value problem (4.2), (4.3).

We choose $N=48$ and $\ell / L=3 / 4$. The location of the roots $\xi_{j}^{(48)}(j=0,1, \ldots, 48)$ of the Hermite polynomial $H_{49}$ is shown in Figure 1.

The numerical time integration of (4.2) has been carried out with the software Mathematica 4.0 that uses the Livermore Solver LSODE. This solver is based upon linear multistep methods and varies dynamically the step size as well as the method's order. So the time steps are chosen automatically in an adaptive way. The results are compared with the exact solution given by (4.5). (In order to evaluate (4.5), we again use Mathematica. For the following discussion, we assume that the spatial numerical integration of (4.5) and the temporal nu- 

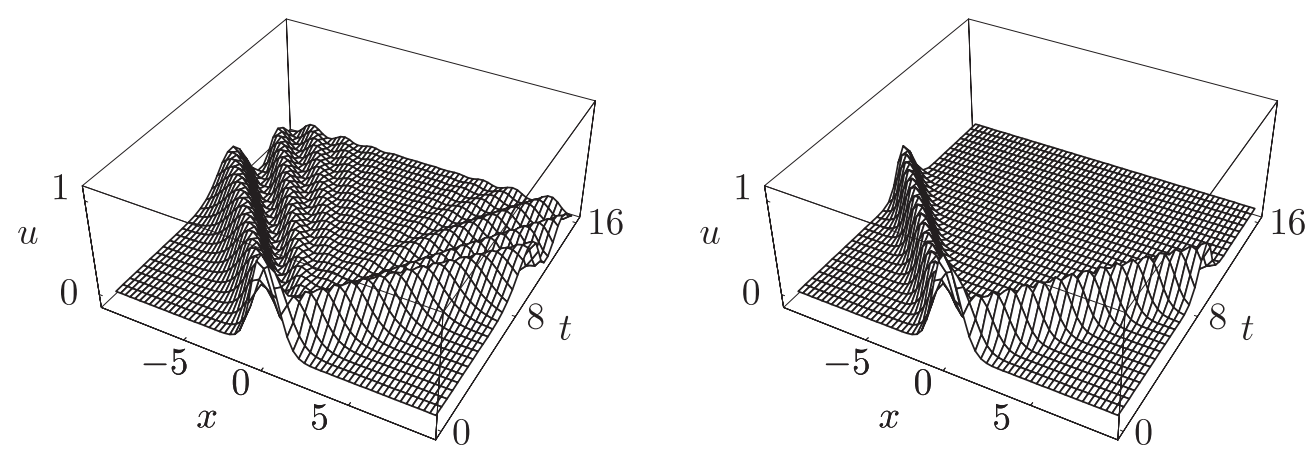

Figure 2. Exact peridynamic (left) and wave (right) solution for Example 4.1.
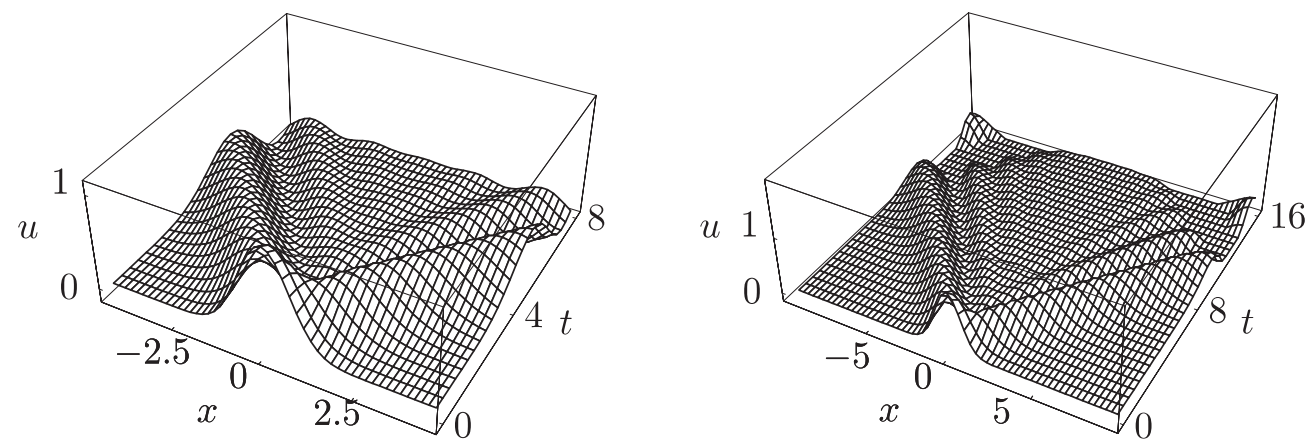

Figure 3. Numerical peridynamic solution for Example 4.1.

merical integration of (4.2) are exact within the resolution of the line thickness. This can be verified by increasing the working precision such that the numerical and graphical results presented do not change.)

The classical wave solution and the peridynamic solution is presented in Figure 2.

Figure 3 shows the numerical solution.

It turns out that the numerical results agree quite well with the analytical results for all points $x \in\left[\xi_{0}^{(48)} \approx-9.1, \xi_{48}^{(48)} \approx 9.1\right]$ if we limit the time of observation up to $t \approx$ 8. Increasing the number of Gauss-Hermite quadrature points extends both the spatial and temporal observation interval. The valid temporal observation period can be also extended if we restrict our attention to points closer to the origin: the computed displacement of the particle located at the origin $0=\xi_{24}^{(48)}$ is a good approximation until $t \approx 14$. For $t>14$, however, the results diverge for all points $x$. In particular, the method presented fails when predicting long-time behavior. This is due to the wave reflection at the artificial boundary that comes into play when approximating the improper integral by an integral over $\left[\xi_{0}^{(N)}, \xi_{N}^{(N)}\right]$ as in (4.1). 


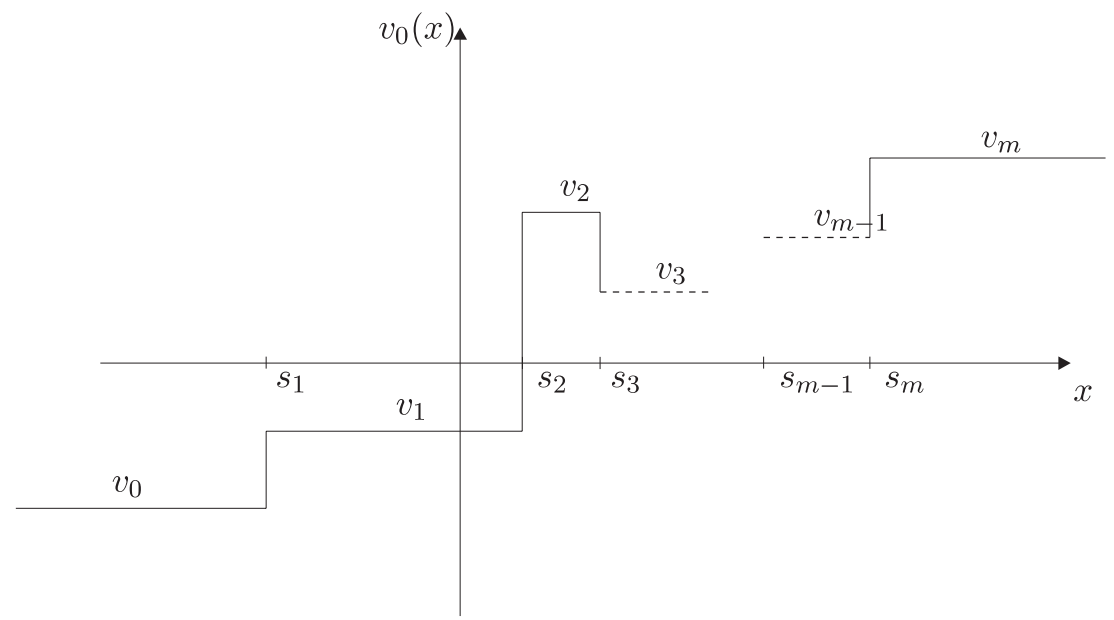

Figure 4. Initial velocity field involving multiple jump discontinuities.

Example 4.2 (Multiple jump discontinuities in the initial velocity field). For $s_{1}<s_{2}<$ $\cdots<s_{m}$ and $v_{0}, v_{1}, \ldots, v_{m} \in \mathbb{R}$ given, we consider the initial displacement and velocity (see also Figure 4)

$$
u_{0}(x) \equiv 0, \quad v_{0}(x)=\frac{v_{0}+v_{m}}{2}+\sum_{i=1}^{m} \frac{v_{i}-v_{i-1}}{2} \operatorname{sgn}\left(x-s_{i}\right) .
$$

Here, sgn is the sign function. The Fourier transforms are given by

$$
\mathcal{F}\left[u_{0}\right](k) \equiv 0, \quad \mathcal{F}\left[v_{0}\right](k)=\left(v_{0}+v_{m}\right) \pi \delta(k)-\imath \sum_{i=1}^{m}\left(v_{i}-v_{i-1}\right) \frac{\mathrm{e}^{-l s_{i} k}}{k},
$$

where $\imath$ denotes the imaginary unit and $\delta$ is again Dirac's $\delta$-distribution. The right-hand side is taken to be zero.

The micromodulus function we wish to investigate reads as

$$
C(x)=\frac{2 E}{\pi \ell^{3}}\left\{\begin{array}{cl}
\frac{\sin \left(\frac{x}{\ell}\right)-\frac{x}{\ell} \cos \left(\frac{x}{\ell}\right)}{\left(\frac{x}{\ell}\right)^{3}} & \text { if } \frac{x}{l} \neq 0, \\
\frac{1}{3} & \text { if } \frac{x}{l}=0 .
\end{array}\right.
$$

and corresponds to the simple dispersion relation

$$
\omega(k)=\frac{c_{0}}{\ell} \begin{cases}|k \ell| & \text { if }|k \ell|<1, \\ 1 & \text { if }|k \ell| \geq 1 .\end{cases}
$$


Again, $\ell$ denotes a length-scale parameter, which characterizes the non-locality, and $c_{0}=$ $\sqrt{E / \rho}$. The homogeneous solution for this material with $u_{0} \equiv 0$ and arbitrary $v_{0}$ is given by

$$
u(x, t)=\frac{\sin \left(c_{0} t / \ell\right)}{c_{0} / \ell} v_{0}(x)+\frac{1}{2 \pi} \int_{-1 / \ell}^{+1 / \ell} \mathrm{e}^{\imath k x} \mathcal{F}\left[v_{0}\right](k)\left(\frac{\sin \left(c_{0} t k\right)}{c_{0} k}-\frac{\sin \left(c_{0} t / \ell\right)}{c_{0} / \ell}\right) \mathrm{d} k
$$

For the above initial velocity, we obtain

$$
\begin{aligned}
\hat{u}(\hat{x}, \hat{t}) & =\frac{\hat{v}_{0}+\hat{v}_{m}}{2} \hat{t} \\
& +\sin (\hat{t}) \sum_{i=1}^{m} \frac{\hat{v}_{i}-\hat{v}_{i-1}}{2}\left(\operatorname{sgn}\left(\hat{x}-\hat{s}_{i}\right)-\frac{2}{\pi} \sin \left(\hat{x}-\hat{s}_{i}\right)-\frac{2}{\pi} \operatorname{Si}\left(\hat{x}-\hat{s}_{i}\right)\right) \\
& +\frac{1}{\pi} \sum_{i=1}^{m} \frac{\hat{v}_{i}-\hat{v}_{i-1}}{2}\left(\left(\hat{x}-\hat{s}_{i}+\hat{t}\right) \operatorname{Si}\left(\hat{x}-\hat{s}_{i}+\hat{t}\right)-\left(\hat{x}-\hat{s}_{i}-\hat{t}\right) \operatorname{Si}\left(\hat{x}-\hat{s}_{i}-\hat{t}\right)\right),
\end{aligned}
$$

where

$$
\hat{x}:=\frac{x}{\ell}, \quad \hat{s}_{i}:=\frac{s_{i}}{\ell}, \quad \hat{t}:=\frac{c_{0} t}{\ell}, \quad \hat{v}_{i}:=\frac{v_{i}}{c_{0}}, \quad \hat{u}(\hat{x}, \hat{t}):=\frac{u(x, t)}{\ell},
$$

and $\operatorname{Si}(x):=\int_{0}^{x} \xi^{-1} \sin \xi \mathrm{d} \xi$ is the integral sine. In the following, we omit the hat and consider only the dimensionless quantities.

Let us assume that $m=2$ with $s_{1}=-1$ and $s_{2}=1$. We then have

$$
K^{(N)}=K_{0}^{\left(N_{0}\right)}+K_{1}^{\left(N_{1}\right)}+K_{2}^{\left(N_{2}\right)},
$$

where $K_{0}^{\left(N_{0}\right)}$ is the approximation of the integral over $\left(-\infty, s_{1}\right), K_{1}^{\left(N_{1}\right)}$ corresponds to $\left(s_{1}, s_{2}\right)$, and $K_{2}^{\left(N_{2}\right)}$ corresponds to $\left(s_{2}, \infty\right)$.

For $K_{0}^{\left(N_{0}\right)}$ and $K_{2}^{\left(N_{2}\right)}$, we again employ the Gauss-Hermite quadrature: Outside the corresponding interval, the integrand is extended by zero and the quadrature points are centred at $s_{1}$ and $s_{2}$, respectively. For a function $f:\left(-\infty, s_{1}\right) \rightarrow \mathbb{R}$, we thus have

$$
\int_{-\infty}^{s_{1}} f(\xi) \mathrm{d} \xi \approx K_{0}^{\left(N_{0}\right)} f:=\sum_{j=0}^{N_{0}} \gamma_{j}^{\left(2 N_{0}+1\right)} \exp \left(\left(\xi_{j}^{\left(2 N_{0}+1\right)}\right)^{2}\right) f\left(s_{1}+\xi_{j}^{\left(2 N_{0}+1\right)}\right)
$$

and a similar representation for $K_{2}^{\left(N_{2}\right)}$, with the roots $\xi_{j}^{\left(2 N_{0}+1\right)}$ of the Hermite polynomial $H_{2 N_{0}+2}$ and $\gamma_{j}^{\left(2 N_{0}+1\right)}$ given by (4.6). The integral over $\left(s_{1}, s_{2}\right)$ is approximated by the composite trapezoidal rule with equidistant quadrature points. For simplicity, we take $N_{0}=N_{2}$ and $N_{1}=2\left(N_{0}+1\right)$. The system (4.2) then consists of $N+1=4\left(N_{0}+1\right)+1$ equations.

Figure 5 shows the analytical and numerical solution $u=u(x, t)$ at the fixed times $t=2,3,4$ with $v_{0}=v_{2}=0, v_{1}=0.5$ and $N+1=101$ quadrature points. For the presentation of the numerical solution, we use piecewise cubic interpolation. 

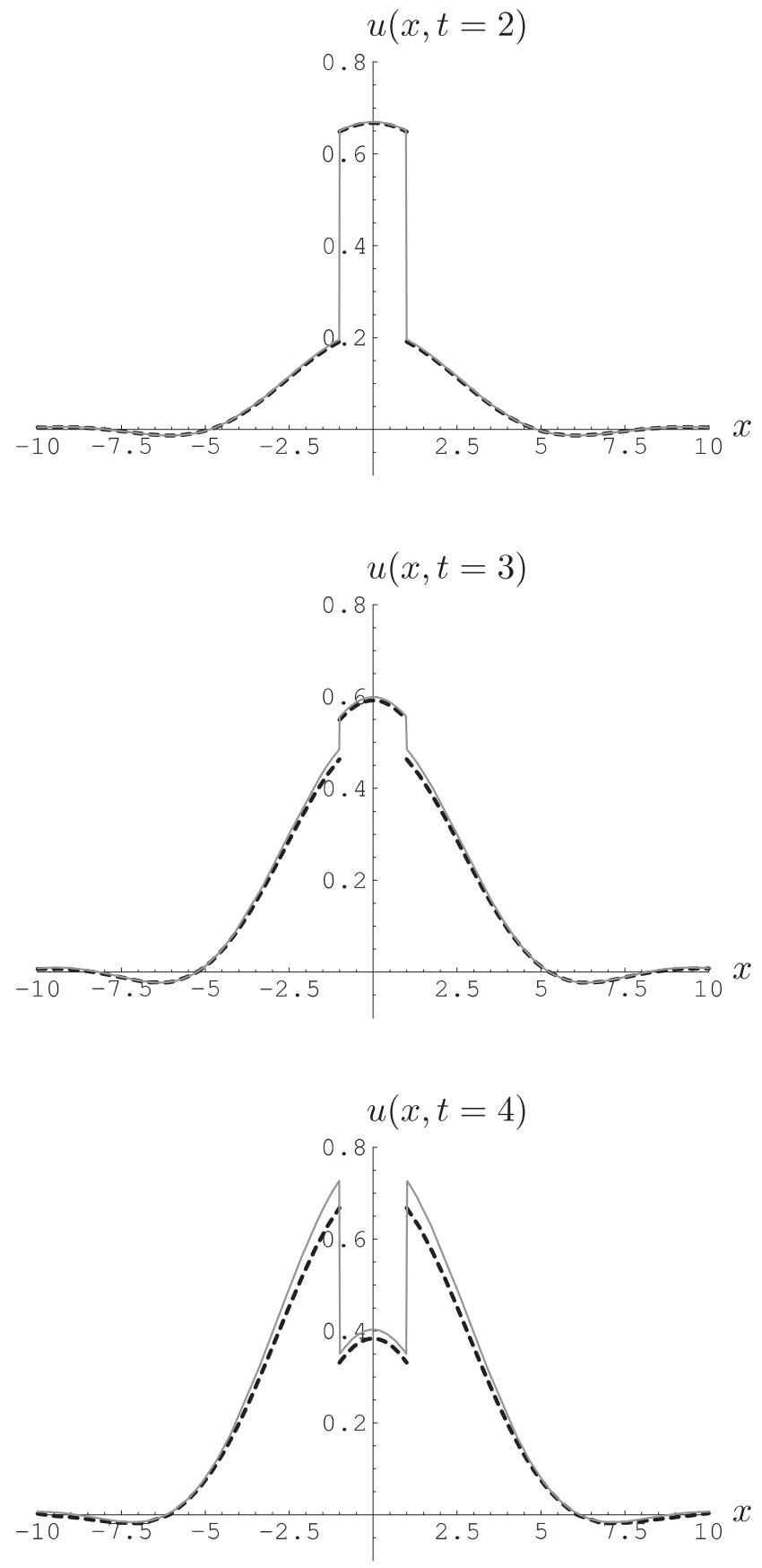

Figure 5. Numerical (dashed lines) compared to analytical (thin lines) results for Example 4.2 at fixed times. 

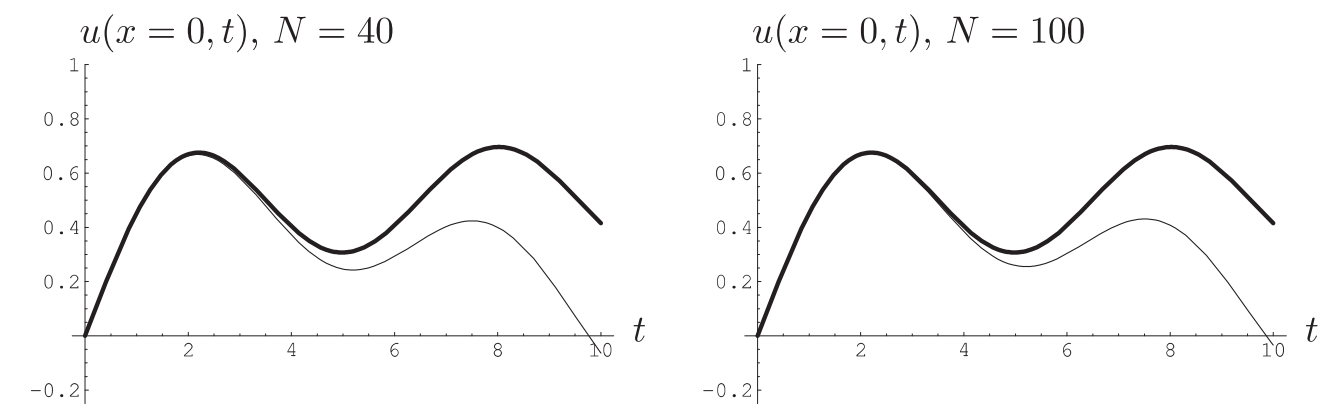

Figure 6. Numerical (thin lines) compared to analytical (thick lines) results for Example 4.2 at $x=0$.

Another way to present the results is to look at a fixed particle at $x=0$ as in Figure 6 . Whereas the left figure shows the numerical results for 41 quadrature points, the right one shows the results for 101 quadrature points. As in Example 4.1, one finds that the numerical results are reasonable only for a finite time interval. The more integration points are taken, the longer this time interval becomes. The convergence seems, however, to be rather slow in this example. In the context of fracture mechanics this is of no concern since the long-time behavior is neither interesting nor could it be described by the same mathematical model (one would expect nonlinearity, plasticity, etc., to play an important rôle here).

The following table shows the CPU-time $T_{C P U}$ needed for the calculation (with an Intel Pentium M 1.4 GHz and $512 \mathrm{MB}$ RAM) with a fixed time interval $[0,10]$ in dependence of the number $N+1$ of quadrature points:

\begin{tabular}{rr}
\hline$N+1[1]$ & $T_{C P U}[s]$ \\
\hline 41 & 92 \\
61 & 408 \\
81 & 1244 \\
101 & 2791 \\
\hline
\end{tabular}

Acknowledgments. This work was supported by a fellowship of the second author within the Postdoc-Programme of the German Academic Exchange Service (DAAD).

\section{REFERENCES}

[1] Silling, S. A. Reformulation of elastic theory for discontinuities and long-range forces. Journal of Mechanics and Physics of Solids, 48, 175-209 (2000).

[2] Silling, S. A., Zimmermann, M., and Abeyaratne, R. Deformation of a peridynamic bar. Journal of Elasticity, 73, 173-190 (2003).

[3] Czycholl, G. Theoretische Festkörperphysik, Springer, Berlin, 2004.

[4] Chen, Y., Lee, J. D. and Eskandarian, A. Atomistic viewpoint of the applicability of microcontinuum theories. International Journal of Solids and Structures, 41, 2085-2097 (2004).

[5] Kunin, I. A. Elastic Media with Microstructure I: One-Dimensional Models, Springer, Berlin, 1982.

[6] Rogula, D. Nonlocal Theory of Material Media, Springer, Berlin, 1982.

[7] Lee, C. T. et al. Non-local concepts and models in biology. Journal of Theoretical Biology, 210, 201-219 (2001). 


\section{E. EMMRICH and O. WECKNER}

[8] Weckner, O. and Abeyaratne, R. The effect of long-range forces on the dynamics of a bar. Journal of Mechanics and Physics of Solids, 53, 705-728 (2005).

[9] Graff, K. F. Wave Motion in Elastic Solids, Dover, New York, 1991.

[10] Tricomi, F. G. Integral Equations, Interscience, New York, 1958.

[11] Appell, J. M., Kalitvin, A. S. and Zabrejko, P. P. Partial Integral Operators and Integro-differential Equations, Dekker, New York, 2000.

[12] Amann, H. Ordinary Differential Equations, de Gruyter, Berlin, 1990.

[13] Emmrich, E. Gewöhnliche und Operator-Differentialgleichungen, Vieweg, Wiesbaden, 2004. 